\title{
Occlusion of the popliteal artery after focused ultrasound treatment - a case report
}

\author{
FP Faccini ${ }^{1}$, JMC Souza $^{2}$, AL Arendt ${ }^{3}$ \\ ${ }^{1}$ Vascular Surgery - Hospital Moinhos de Vento and Instituto de Cardiologia, Porto Alegre, Brasil \\ ${ }^{2}$ Clínica Angiovasc, Aracaju, Sergipe, Brasil \\ ${ }^{3}$ Vascular Surgery - Hospital Moinhos de Vento and Instituto de Cardiologia, Porto Alegre, Brasil
}

submitted: Jun 20, 2019, accepted: Jul 2, 2019, EPub Ahead of Print: Jul 3, 2019, published: Jul 7, 2019

Conflict of interest: None

DOI: 10.24019/jtavr.65 - Corresponding author:Dr. Felipe Puricelli Faccini, felipefaccini@yahoo.com, fpfaccini@gmail.com

(C) 2018 Fondazione Vasculab impresa sociale ONLUS. All rights reserved.

\begin{abstract}
The use of focused ultrasound and highintensity focused ultrasound (HIFU) is common in physical therapy and medical procedures. The ultrasound produces heat and can be used to treat several diseases. We report a case of a 49-year-old male that had a knee injury after mild trauma. The patient started physical therapy sessions to treat the injury. Ipsilateral calf claudication started a few hours after the focused ultrasound was used to heat the popliteal area progressing to symptoms of acute arterial occlusion in two weeks. We started anticoagulation and confirmed popliteal thrombosis with duplex scan and computed tomography. The 5 years follow up showed good clinical recovery and recanalization of the artery. This case reminds us that the focused ultrasound should not be considered harmless and life-threatening complications may occur. The inadvertent heating of neighboring structures and recanalization of the heated vessel may happen.
\end{abstract}

Keywords High intensity focused ultrasound, Acute arterial occlusion, Popliteal artery occlusion, Thermoablation, Recanalization

\section{Introduction}

Focused ultrasound and High-Intensity Focused Ultrasound (HIFU) have been increasingly used in vascular surgery to control hemorrhage and to produce thermal damage in vessels when it is desirable. In the mid twentieth century various conditions were considered possibilities for using the mechanism of tissue heating ${ }^{1}$. Over the following decades, scientific advances allowed the development of better methods for effective treatment of several disease. Since the 1970's, the use of therapeutic ultrasound was established for kinesiotherapy. The continuous ultrasound waves produce localized heating and consequently protein denaturation causing thermal ablation ${ }^{2}$. The more the power the more the likelihood and magnitude of bioeffects ${ }^{1}$. The use for hemostasis of punctured arteries has achieved promising results in animal studies ${ }^{3,4}$. Spleen and liver hemostasis have also been successfully achieved using HIFU in animals ${ }^{5-7}$. The long term effects of most Focused Ultrasound and HIFU treatments are still unknown and knowledge about complications of its use is scarce. The ultrasound technology has been proved to perform thermal ablation on veins ${ }^{8}$ and recently the technique is being performed to obtain thermal ablation of the saphenous and varicose veins. The focus of the ultrasound beam is pivotal to obtain the expected results without complications. Although the ultrasound is considered safe, the produced heat can cause injury to structures not intended to be damaged ${ }^{1}$, like arteries and nerves that usually are anatomically close to veins. This possibility was considered by Henderson et al and described in an ex-vivo study 9 .

The use of focused ultrasound in physical therapy is well established and complications are rare, although the level of efficacy is modest and patient benefits are 
uncertain $^{1}$. We report a case of popliteal artery thrombosis after focused ultrasound therapy. Ultrasound biophysics, complications and uses in vascular surgery of both focused and high-intensity focused ultrasound are discussed.

\section{Case report}

A 49-year-old male presented to the clinic with left leg pain, coldness and paleness with no sensory loss, no muscle weakness and audible arterial and venous Doppler. We noticed an unusual coloring at the skin of the popliteal region only, not related to areas of ischemia. Medical history showed a mild fall injury to the knee 4 months before and focused ultrasound during physical therapy sessions for bone healing two weeks before the vascular appointment. The patient referred intermittent claudication and leg coldness starting a few hours after a session of physical therapy in which the focused ultrasound was used.

The progression to severe symptoms of ischemia have happened 2 weeks later. The duplex scan (Figure 1) showed popliteal thrombosis and computed tomography showed $8 \mathrm{~cm}$ of unusual obstruction of the popliteal artery at the knee region (Figure 2). As we did not operate the patient, no histologic sample is available. Initially, we considered that the cause of arterial occlusion was either arterial trauma or burning from the ultrasound used to heat the region. Literature review about arterial damage after knee injury suggests that the onset of arterial occlusion was late to blame trauma alone. Most literature reports and case series present occlusion in the first hours or few days after trauma ${ }^{10}$ and our patient presented occlusion more than 4 months after trauma.

Although, we consider that the trauma might have been an adjunct factor due to the possibility that a hematoma change the position and mobility of the artery. Embolism and atherosclerosis were excluded by careful cardiac and arterial evaluation. The symptoms prior to the focused ultrasound were articular pain depending on the position of the knee and changed completely after the session.

We started anticoagulation and close observation. The symptoms of acute ischemia improved in a few hours. After the improvement of the first days we suspended heparin and started clopidogrel. The patient remained with intermittent claudication for long distances after 1 week until now and continued stable for the last 5 years.

We decided to avoid invasive treatment with patient's agreement. We do not have large experience with burning arterial lesions and decided to avoid further treatment because the symptoms were stable and not bothering the patient. One year after the treatment the patient had another CT scan that showed complete recanalization of the artery with 2 narrowing areas of $2 \mathrm{~cm}$ each (Figure 3 ). No surgical or endovascular treatment was performed. Concerning the equipment and protocol used to perform the session of kinesiotherapy with focused ultrasound, they are pivotal to better understand the damage. The probe used in the knee region had a frequency of $1,0 \mathrm{MHz}$ using continuous ultrasound and surface power density of $2.0 \mathrm{w} / \mathrm{cm}^{2}$. The patient told us the duration of ultrasound was close to 30 minutes but we could not obtain the complete details.

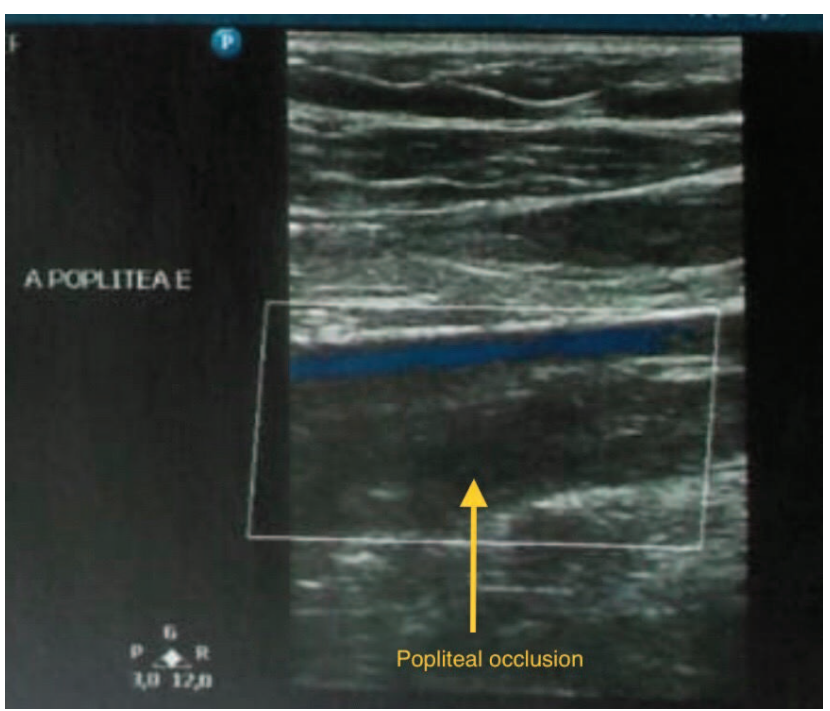

Figure 1 - No flow with Color Doppler in the popliteal artery at the first access.

\section{Discussion}

Ultrasonic energy can provoke biological effects creating heat that can be used to treat diseases. Additionally, ultrasound causes cavitation with microbubbles, increase of gas body activation, radiation force (mechanical stress), and other undetermined non-thermal processes ${ }^{1,11}$. All these effects create desired and undesired biological responses during the medical application of ultrasound.

The diseases in which ultrasound is used for treatment are kidney stones with the lithotripsy; lens removal with the phacoemulsification; bone fracture with the low intensity US; plantar fasciitis and epicondylitis with the extracorporeal shockwaves therapy; uterine fibroid ablation, glaucoma, laparoscopic tissue ablation or laparoscopic or open surgery - all this with HIFU, among others uses ${ }^{1}$.

The rate of serious complications after the focused ultrasound is considered very low and the procedures are common, although no large studies on safety are available ${ }^{1}$. Serious complications as injury to artery, nerves or veins are considered rare. We have not found any report in the literature of popliteal artery occlusion caused by ultrasound. 


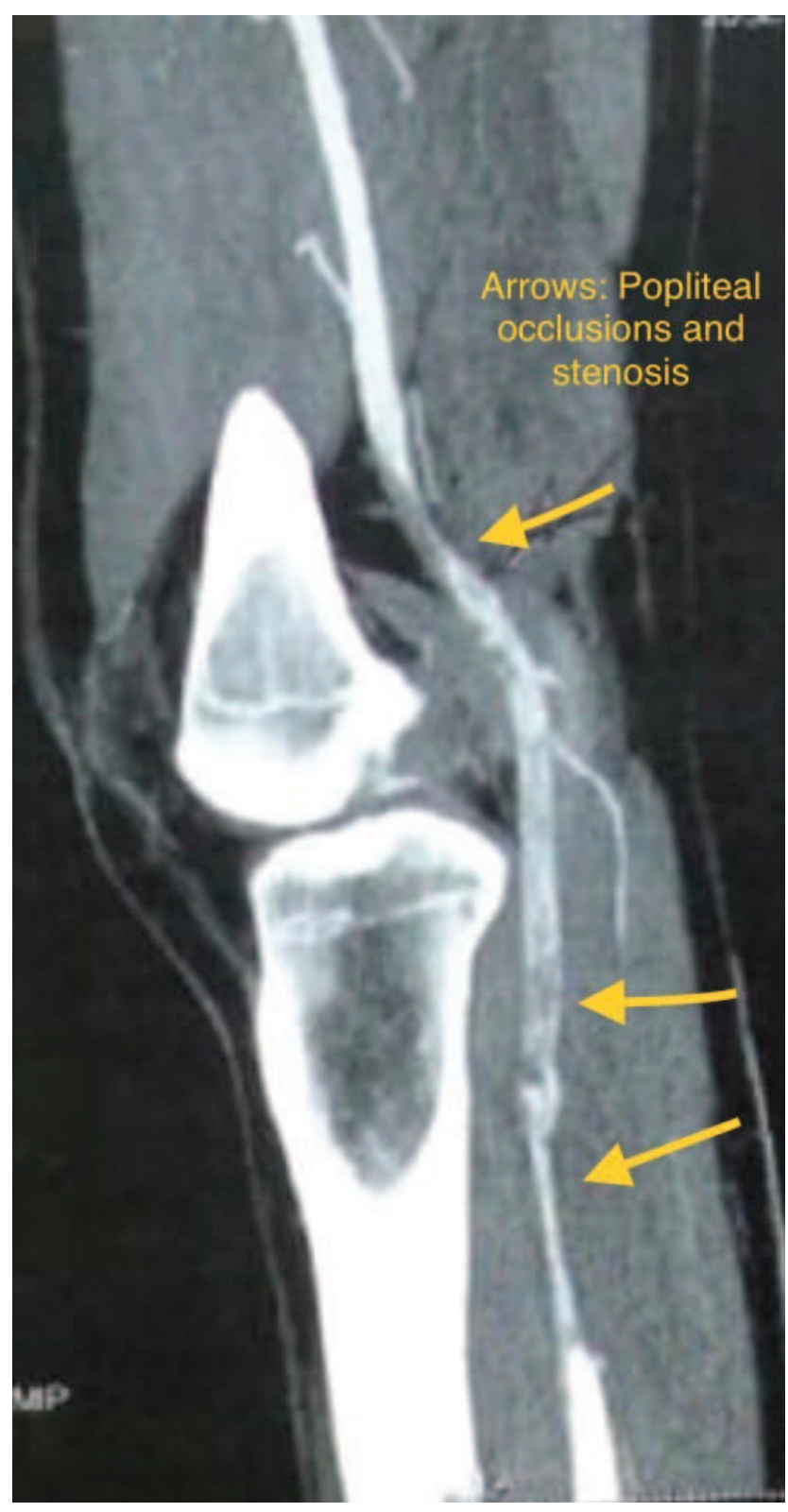

Figure 2 - Computed tomography image confirming the obstruction at the popliteal artery.

This is probably an extremely rare complication because most focused ultrasounds are not used near important arteries or structures. In our isolated clinical case we have noticed that the symptoms in the occlusion after ultrasound were not abrupt as in most arterial occlusions, maybe because the processes developed with progressive tissue edema before the full occlusion. The use of HIFU for venous ablation has been studied for a few years. Henderson et al described a portable equipment that successfully ablated veins in an ex-vivo study ${ }^{9}$. Experimental studies have also searched for ways to focus the damage to specific

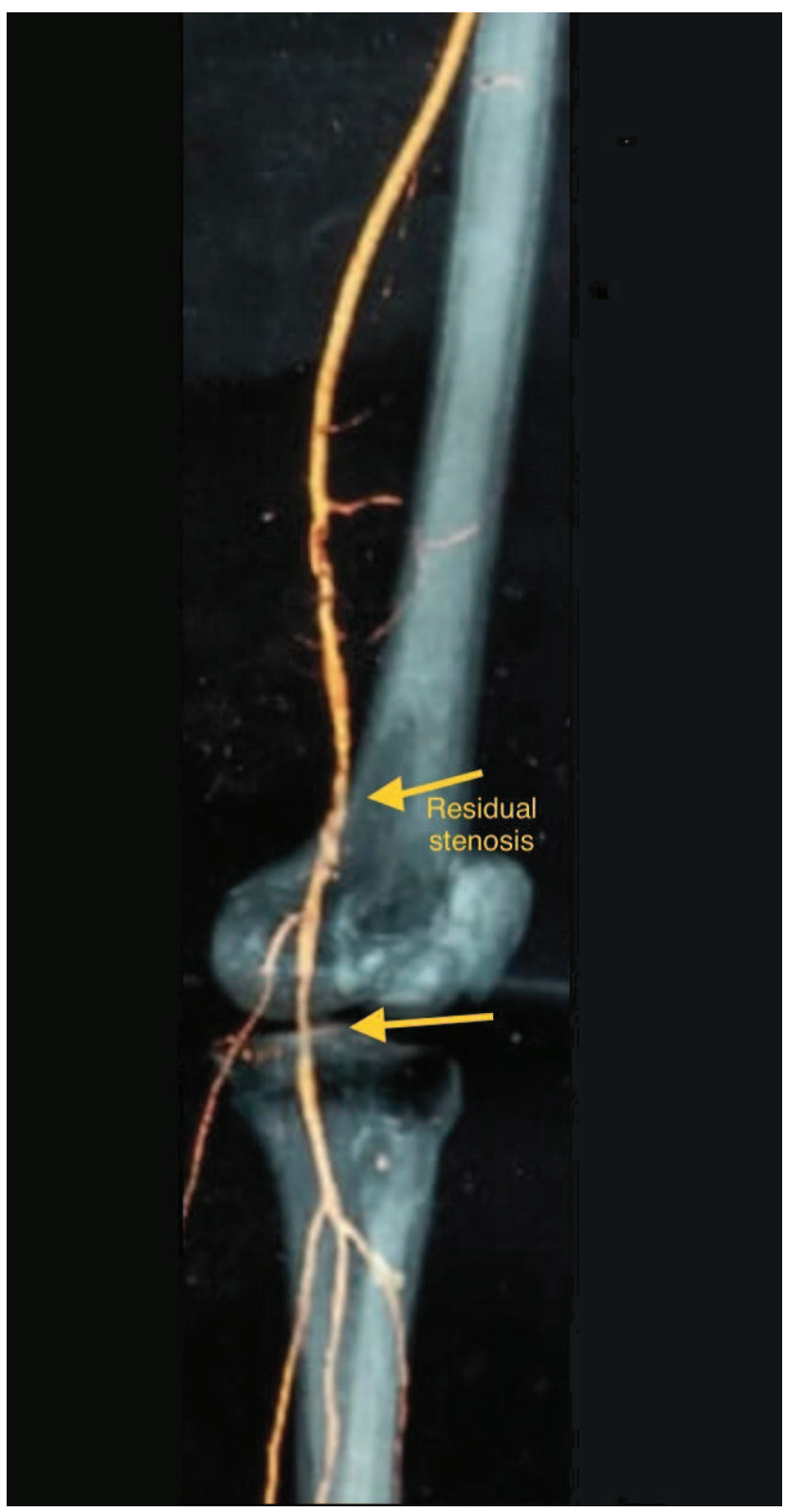

Figure 3 - Computed tomography 1 year after the first examination with focal stenosis at knee level.

vessels by echo contrast and beam control, but the results are still initial ${ }^{12}$. One experimental study in rabbits showed that the HIFU can close by thermal ablation veins as large as $6 \mathrm{~mm}$ with 15 days of follow up ${ }^{13}$. Obermayer has recently shown that HIFU can cause thermal ablation of varicose veins, perforators and great saphenous veins. No long term results are available to ensure the safety and benefit for the patients using the method ${ }^{14}$. In our case, the patient had complete occlusion of the popliteal artery after ultrasound and full recanalization in months, this might 
happen with the veins closed. We expected a definitive arterial occlusion, but time proved we were wrong.

High intensity focused ultrasound HIFU is newer than focused ultrasound and the procedures are still being made under controlled trials in the majority of fields. There is no available data for the safety of HIFU in vascular procedures. Obermayer treated varicose veins and reported 4 legs out of 50 with dysesthesias suggesting damage to nerves. No deep vein thrombosis, pulmonary embolism or arterial damage were reported in the initial cases ${ }^{14}$. Our case reminds us that the energy created by ultrasound can damage neighboring structures as well as the treated vessel.

\section{Conclusion}

In conclusion, the focused ultrasound and highintensity focused ultrasound (HIFU) are important

\section{References}

1) Miller DL, Smith NB, Bailey MR, Czarnota GJ, Hynynen $\mathrm{K}$, Makin IRS, et al. Overview of therapeutic ultrasound applications and safety considerations. J Ultrasound Med [Internet]. 2012 Apr;31(4):623-34. Available from: https://www.ncbi.nlm.nih.gov/ pubmed/22441920 at the date of Jun 20, 2019.

2) Webb H, Lubner MG, Hinshaw JL. Thermal ablation. Semin Roentgenol [Internet]. 2011 Apr;46(2):133-41. Available from: https:// doi.org/10.1053/j.ro.2010.08.002 at the date of Jun 20, 2019.

3) Zderic V, Keshavarzi A, Noble ML, Paun M, Sharar SR, Crum LA, et al. Hemorrhage control in arteries using highintensity focused ultrasound: A survival study [Internet]. Vol. 44, Ultrasonics. 2006. p. 46-53. Available from: https://doi.org/10.1016/ j.ultras.2005.08.002 at the date of Jun 20, 2019.

4) Martin RW, Vaezy S, Kaczkowski P, Keilman G, Carter S, Caps M, et al. Hemostasis of punctured vessels using Doppler-guided high-intensity ultrasound [Internet]. Vol. 25, Ultrasound in Medicine \& Biology. 1999. p. 985-90. Available from: https://doi.org/10.1016/ s0301-5629(99)00027-7 at the date of Jun 20, 2019.

5) Noble ML, Vaezy S, Keshavarzi A, Paun M, Prokop AF, Chi EY, et al. Spleen Hemostasis Using High-Intensity Ultrasound: Survival and Healing [Internet]. Vol. 53, The Journal of Trauma: Injury, Infection, and Critical Care. 2002. p. 1115-20. Available from: https:// doi.org/10.1097/00005373-200212000-00014 at the date of Jun 20, 2019.

6) Burgess S, Zderic V, Vaezy S. Image-guided acoustic hemostasis for hemorrhage in the posterior liver. Ultrasound Med Biol [Internet]. 2007 Jan;33(1):113-9. Available from: https:// doi.org/10.1016/j.ultrasmedbio.2006.07.025 at the date of Jun 20, 2019.

7) Vaezy S, Vaezy S, Starr F, Chi E, Cornejo C, Crum $\mathrm{L}$, et al. Intra-operative acoustic hemostasis of liver: production of a homogenate for effective treatment. Ultrasonics [Internet]. 2005 Feb;43(4):265-9. Available from: https://doi.org/10.1016/ j.ultras.2004.07.002 at the date of Jun 20, 2019.

8) Jiang C-P, Wu M-C, Wu Y-S. Inducing occlusion effect in $\mathrm{Y}$-shaped vessels using high-intensity focused ultrasound: finite techniques that can be used in a variety of medical conditions. The use of the ultrasound wave is generally safe but serious complications might happen and we should always consider that the neighboring structures might be affected by the heating effect or non-thermal effects. Therefore, It is important to accurately determine the location and control the treatment zone with the ultrasound system ${ }^{8}$. The possibility to treat venous disease by ultrasound is exciting and should be deeply studied. Although, we should keep in mind that undesirable and even life-threatening conditions can occur. Safety studies on the new techniques are imperative to validate their use both in terms of safety and long-term benefit of the treatment. In our case we observed both the undesirable occlusion of an important artery and its fast recanalization without intervention

element analysis and phantom validation. Comput Methods Biomech Biomed Engin [Internet]. 2012;15(4):323-32. Available from: https:// doi.org/10.1080/10255842.2010.535521 at the date of Jun 20, 2019.

9) Henderson PW, Lewis GK, Shaikh N, Sohn A, Weinstein AL, Olbricht WL, et al. A portable high-intensity focused ultrasound device for noninvasive venous ablation. J Vasc Surg [Internet]. 2010 Mar;51(3):707-11. Available from: https://doi.org/10.1016/ j.jvs.2009.10.049 at the date of Jun 20, 2019.

10) Kim J-W, Sung C-M, Cho S-H, Hwang S-C. Vascular injury associated with blunt trauma without dislocation of the knee. Yonsei Med J [Internet]. 2010 Sep;51(5):790-2. Available from: https://doi.org/10.3349/ymj.2010.51.5.790 at the date of Jun 20, 2019.

11) O'Brien WD Jr. Ultrasound-biophysics mechanisms. Prog Biophys Mol Biol [Internet]. 2007 Jan;93(1-3):212-55. Available from: https://doi.org/10.1016/j.pbiomolbio.2006.07.010 at the date of Jun 20, 2019.

12) Tokarczyk A, Rivens I, van Bavel E, SymondsTayler R, ter Haar G. An experimental system for the study of ultrasound exposure of isolated blood vessels. Phys Med Biol [Internet]. 2013 Apr 7;58(7):2281-304. Available from: https:// doi.org/10.1088/0031-9155/58/7/2281 at the date of Jun 20, 2019.

13) Barnat N, Grisey A, Lecuelle B, Anquez J, Gerold B, Yon S, et al. Noninvasive vascular occlusion with HIFU for venous insufficiency treatment: preclinical feasibility experience in rabbits. Phys Med Biol [Internet]. 2019 Jan 7;64(2):025003. Available from: https://doi.org/10.1088/1361-6560/aaf58d at the date of Jun 20, 2019.

14) Obermayer A. Ultrasound-Guided High-Intensity Focused Ultrasound Extracorporeal Treatment of Superficial Lower Limb Veins: Preliminary Results and Method Description. Journal of Vascular Surgery: Venous and Lymphatic Disorders [Internet]. 2018;6(4):556-7. Available from: https://www.jvsvenous.org/action/showCitFormats? pii=S2213-333X

$\% 2818 \% 2930168-9 \&$ doi $=10.1016 \% 2$ Fj.jvsv.2018.05.007 the date of Jun 20, 2019. 